\title{
Protection Strategy Exploration for the Computer Network Information Security in the Big Data Age
}

\author{
Liang Xue, Juan Yang, Weiguo Fan \\ Suzhou Polytechnic Institute of Agriculture, Suzhou Jiangsu,215000,China
}

Keywords: Big Data Age, Computer, Network Information, Safety.

\begin{abstract}
With the popularization and application of internet, human society enters the information-based society in an all-round way, computer has gradually become an indispensable part in people's daily life, work and study, it brings great convenience for people as well as some corresponding potential safety hazards. Especially under the current background of the big data age, the internet is inundated with a large amount of data information, these data information has a wide range, opening features of the internet makes the computer network information security problems become more and more serious, a lot of online frauds and information leakage problems happen frequently, which seriously restricts the stable development of the society and has received a widespread attention. In the current big data age, computer stores a large amount of information, how to effectively avoid the computer network information risk, protect the data security, becomes our primary work direction. Therefore, this paper mainly analyzes the protection strategy for computer network information security in the big data age, according to the practical situation to put forward reasonable countermeasures for the existing problems, thus to ensure the network information security and promote the harmonious and stable development of the society.
\end{abstract}

\section{Introduction}

Under the current background, computer is gradually popularized in numerous households and becomes an essential part of people's daily life. [With] At the same time, computer networks get continuous development and improvement,applications and promotion in various industries have achieved a considerable success, people are highly depend on computer network information, which also correspondingly brings a serious network security threats. Computer network is a system discipline, for its own opening features, it not only includes computer communication technology, but also includes information security technology. Especially under the background of the current big data age, data information on the internet is complicated and with large range,a lot of users' important private data is stored in the computer, which is very vulnerable to be attacked, steal or tamper the important data will bring serious potential security hazards to the users. Therefore, it is very necessary to carry out research for computer network information security in the big data age, which helps to provide a reference for subsequent theoretical research and practical work.

\section{Summary of the computer network information security in the big data age}

With the continuous improvement of computer technology and network technology and the gradual in-depth of the information construction, the big data age comes quietly, and receives widespread concern and attention from all walks of life. Computer popularizes in thousands of households, penetrating together with Internet technology into many industries around the world, so to say, the higher the informatization degree is, the faster the era develops, thus marks the arrival of the big data age. Under the background that the big data age arrives, application of computer networks popularizes in many industries and fields, constructing a digital world through the storage and share 
of data information, people's angle for the objective world has been changed, corresponding countermeasures also changed, so to speak, the society has gone through huge changes under the background of the big data age ${ }^{[1]}$.

The arrival of big data age mainly bases on the widely application and popularization of computer network technology, which has a outstanding acceleration in all kinds of industries, it has an incomparable place in the life and production, therefore, under the current background, it is very necessary to strengthen the security protection of computer network information. As a new technology, computer network technology is the main power that stimulates the development of current society, under the background of big data age, the range of computer network information security is gradually enlarging, diversified technology can bring the computer network a certain security, while when the computer network is working, for its opening features, it is easy to be attacked by lawbreakers to steal important data information, which threats the user's privacy ${ }^{[2]}$. Therefore, in view of these potential risks of computer network information, we should establish specified protection system for the computer network information, continue to complete and innovate to propose reasonable countermeasures through analyzing its potential safety risks to meet the protection demand for the computer network information security under the background of big data age, to promote the harmonious and stable development of the society.

\section{Risk factors of the computer network information security in the big data age}

\section{Natural disaster}

Computer has fixed mechanical equipment, which cannot effectively resist the influence and destroy from the outside, once natural disaster happens, it may cause earthquake, fire and water disaster and lighting and thunder threats, all of these will influence the computer equipment, it is difficult to guarantee the safety of the computer equipment. Therefore, the computer equipment is very fragile, has no ability for resisting natural disasters, which is one of the main factors that influence the security of computer equipment under the big data age background.

\section{Its own opening features}

Because of its own features, the computer network gradually shows its initial opening features during its wide application, it also its opening features causes the fragility of the computer internet system, that it is hard to resist attack from outside ${ }^{[3]}$. Based on its opening features, the computer network users need to apply the TCP/IP suite, with low security and big potential security problems, in the practical operation, it is hard to effectively resist attack from outside, and hard to provide better service to meet actual work demand. Therefore, its own opening features cause the threat on the network security, which leads to the weak foundation, and becomes one of the main factors.

\section{Operational errors}

Operational error mainly refers to that when users operates the computer, because they are lack of relevant knowledge, they completely rely on their own experience and subjective consciousness, thus easy to leave potential risks for the computer network information. During the actual operating process, because of the opening features of the internet, users' different level of operation security sense and skill, there might be obvious difference in the operation, for some operation with potential risks, users are difficult to recognize, thus users' operation problem will be one of the main factors that influence the computer internet information security ${ }^{[4]}$.

\section{Hacker attack}

Hacker attack is now one of the main factors that influence computer network information security, meanwhile, it is the most typical factor. Hacker attack mainly can be divided into two aspects, one is active attack, this kind of attack is consciously and pointed to users' computer, it will cause breakdown of the system, a large amount of important data will be stolen and tampered; the other one 
is positive attack, mainly are interception and crack of the objected information, in general case, this kind of attack will not affect the normal operation of the system, but will cause the steal of private data. Though these two attacks have essential difference, but at last they will cause different influences for users, will cause large amount of data loss, even bring serious threat to the computer network security. This kind of spite attack will not only cause the loss of users' private data, but also will cause transfer compensation of the computer network information, finally leads to the breakdown of the system, which will influence social life and production, cause serious economic loss.

\section{Computer virus invasion}

Under the current background of big data age, the opening features of computer network information and users' wrong operation can easily bring virus invasion to the computer to affect the computer network information security. After the virus invading into the computer, it will drill the computer bug, change the system, or steal users' private data,thus affects the normal usage of the computer. Because of the feature of witness hiding of the computer virus, once it invade into users' computer system, the infectious virus has strong destructiveness, breaks the computer system, and cause huge harms to the internet data security ${ }^{[5]}$. In terms of the internet virus broadcasting carrier, mainly through software, hardware and optical disc to bring virus into the system, for some virus with strong destructiveness, they have outstanding influence on the normal operation the computer system, for example, the Nimaya and $\mathrm{CIH}$ virus popular several years before, which swept through the internet at that time, cause large amount of data loss and system breakdown, brought a serious influence on the computer network information, which is not benefit for the harmonious and stable development of the society.

\section{Junk information}

Junk information is mainly spread through email and news, during its spreading process, these junk information has a certain mandatory characteristic, it will infuse spams mandatory, among which are mainly commercial advertisements. While the information stolen are mainly caused by the software invasion of the law breakers, they break down the computer system through the computer virus invasion to steal users' information, cause the breakdown of system, seriously threats users' information security, which is not good for the normal operation of computer internet. Therefore, junk information in the computer system and information stolen influenced the computer network information security.

\section{Protection strategy of the computer network information security in the big data age}

Computer network information security is a main obstructive factor for the current information construction, it is easy to cause large amount of important data information loss, if company lost its important data, it is easy to bring opportunity for its competitor, and will become inactive in the fierce marketing competition, will restrict its healthy and sustainable development; if people lost his/her important data, it may cause serious influence and destroy personal reputation on a certain degree or leads to financial loss.

\section{Pay attention to the account security}

Under the background of current big data age, the security protection of computer network information should consider from multiple angles to analyze the factors that influence computer network information security, pay attention the the management of account security. Account information includes many forms, like bank account, game account, computer system account, email account and so on, effectively manage these private accounts should to improve the security protection sense for account security, try to set up complex passwords, that can avoid the leakage of the passwords, also can set special symbols in the passwords, thus can avoid same passwords, change 
passwords periodically, thus can avoid account leave network trace and be to stolen by the law breakers.

\section{Network firewall technology}

Network firewall technology is a means mainly for network visiting, is one of the main means to prevent invasion of law breakers, prevent outside people to get into the inner system of computers, on a certain degree, it can provide security protection for computer network system, and provide better network environment. Network firewall technology is to realize data transformation on the basis of network security, determine whether the outside visiting get through or not through the operation of security procedure. Based on its different type, the network firewall technology can be divided as multiple technologies like agent type, testing type and address translation type. Though with different technology, all the network firewall technology are to make restriction for outside users, prevent all the potential threats of the computer network form the outside, to provide security protection for the normal operation of the network.

\section{Use anti-virus software}

Computer network information security is influenced by many kinds of objective factors, installing anti-virus software is mainly to cooperate firewall to inspect the potential risks, it has a very outstanding effects in the real application, and is widely used in the computer system. The function of anti-virus software is to inspect and kill virus that are already found, to check whether the computer system is attacked or not, to protect the security of the network information. When using the anti-virus software, should pay attention to its upgrading, continue to update the system mend, improve the kill ability of the software, provide more sufficient protection for the computer network information security.

\section{Network monitoring}

In recent years, the application of network monitoring has achieved good effects, it gradually gains people's welcome and like, corresponding detection technology also achieved innovation and perfection, it can effectively detect whether there is attack from the law breakers. This attack detection technology mainly includes signature analysis and statistical analysis, signature analysis is to check whether there is bugs that influence system security in the current system. Statistical analysis is to check whether the computer system can operate in the normal way, analyze the stable operation situation thus to detect the computer system. In a manner of speaking, network monitoring technology provides a better technical protection for the computer network information security.

\section{Data preservation and encryption}

Because of the opening features of the internet, important data are easy to be influenced by the outside world during its transmission and storage process, which may lead to the phenomenon that data are stolen or tampered. Protection of data security is the important content of protection for computer network information security under the big data age, data storage emphasizes data encryption, this data encryption technology can effectively improve data security and prevents it to be stolen and tampered. Link encryption is a normal way of data encryption, mainly emphasizes safe transmission in the link transmission, improve data information security by using secret key to protect its security.

\section{Conclusion}

In conclusion, under the background of big data age, computer network information security faces serious challenge, because of its own opening features, it is easy to be attacked by law breakers in the practical application, which affects data security, cause the loss of important data, cause immeasurable effects and loss. Therefore, for the transmission, storage and communication of computer network information, establishing a more safe and reasonable computer network operation 
environment can effectively strengthen computer information security and construct a healthier and harmonious network environment.

\section{References}

[1] Ruxian Guli.Abu Dure Xiti. Research on the Computer Network Security and Protection Strategy in the Information Age, Computer Knowledge and Technology,2015,11(06):30-31+36.

[2] Chen Jian. Exploration on Computer Network Security and Protection Strategy, Electric Technology and Software Project, 2013,12(23):242.

[3] Wang Chao. Research and Design of Invasion Detection System in the Computer Network Security, University of Electronic Science and Technology of China,2013.

[4] Lu Xiaorui. Research on Computer Network Security and Protection Strategy, Electric Production,2013,24(13):115-116.

[5] Zhu liang. Computer Network Information Management and Its Protection Strategy, Computer Knowledge and Technology,2012,16(18):4389-4390+4395. 